\title{
Frequência de consumo de biscoitos "Maria" por escolares de Fortaleza - Ceará: Subsídios para a eleição do produto para fortificação com ferro
}

\begin{abstract}
Dora Neumann ${ }^{1}$, Maria do Carmo Passos Rodrigues ${ }^{2}$, Dalton Francisco de Andrade ${ }^{3}$
O objetivo da pesquisa foi avaliar a freqüência de consumo de biscoitos "Maria" por escolares visando à eleição deste alimento como veículo para fortificação com ferro e a seleção de escolares para avaliação sensorial do produto fortificado. Participaram da amostra 727 alunos do ensino fundamental de 18 escolas da rede municipal de Fortaleza-CE. Foi aplicado um questionário de freqüência de consumo. A seleção de alunos obedeceu a um plano amostral estratificado, no qual os estratos foram constituídos pelas seis secretarias regionais às quais as escolas estavam vinculadas, sendo as classes de $2^{\mathrm{a}}$ a $8^{\mathrm{a}}$ séries do ensino fundamental as unidades primárias de seleção. Para o cálculo da freqüência média de consumo atribuiu-se uma ponderação para os itens de freqüência de: i) 4 - diariamente (consumo elevado); ii) 3 duas a três vezes por semana (consumo moderado); iii) 2 - uma a duas vezes por mês (consumo baixo); iv) 1 - uma a duas vezes por semestre (consumo raro); v) 0 - nunca (nulo). Foi realizada análise descritiva dos dados de consumo por sexo, idade e regional, estabelecendo-se uma freqüência de consumo média para estes parâmetros por meio de modelo linear geral, aplicando-se teste estatístico de hipóteses ao nível de significância de $5 \%$. Aproximadamente $60 \%$ dos escolares de ambos os gêneros e faixas etárias informaram consumir o produto diariamente ou 2 a 3 vezes por semana. Uma estimativa única para representar a média para ambos os gêneros foi de 2,34 (DP= 0,04), e para todas as faixas etárias foi de 2,66 (DP= 0,04$)$ sugestiva de consumo moderado. Os resultados revelaram a adequação do produto como veículo para fortificação com ferro, sob o aspecto de consumo e a aptidão do grupo como consumidores potenciais, para a avaliação sensorial do mesmo.
\end{abstract}

Palavras-chave: alimentação escolar, biscoitos, freqüência de consumo.

\section{Frequency of consumption of biscuit "Maria" for school students from Fortaleza, Ceara state, aiming the election of the product to be fortified with iron}

\begin{abstract}
The purpose of this research was to evaluate the consumption frequency of Maria biscuits for school students aiming at choosing this food as a vehicle for iron fortification and the selection of school pupils for sensorial evaluation of the fortified product. Seven-hundred and twenty-seven pupils from the primary level of 18 schools from the municipal school system of Fortaleza, Ceará, participated of this sampling filling out a questionnaire of consumption frequency. The selection of pupils obeyed a stratified data plan, in which the strata were composed of the six regional educational public districts, selecting the second to eighth grades of elementary education classes. By calculating the mean consumption frequency a weighted estimate of the frequency was obtained: i) 4 - daily (over consumption); ii) 3 - twice to three times per week (moderate consumption); iii) 2 - once to twice per month (low consumption); iv) 1 - once to twice per semester (rare consumption);
\end{abstract}

\footnotetext{
${ }^{1}$ Prof ${ }^{a}$ do Depto. de Nutrição - Centro de Ciências da Saúde - Universidade Federal do Pará - Mestre em Tecnologia de Alimentos Praça Camilo Salgado No 1 - Bairro Umarizal - Belém - PA CEP: 66.050.060 - Fone: (91)32152350 - Fax: (91) 32429412 e-mail: neumann@fea.unicamp.br, doraneumann@ig.com.br

${ }^{2}$ Prof ${ }^{a}$ Dra do Depto. de Tecnologia de Alimentos - Universidade Federal do Ceará - Fortaleza-CE e-mail: frutos@ufc.br

${ }^{3}$ Prof. Dr. Titular do Depto de Informática e Estatística - Universidade Federal de Santa Catarina - e-mail: dandrade@inf.ufsc.br
} 
v) 0 - never (nullity). A Descriptive analysis carried through with the frequency consumption data by gender, age and regional office was made establishing an average frequency of consumption for these parameters using a general linear model, applying a hypotheses statistics of $5 \%$ significance level. Approximately $60 \%$ of the school pupils from both genders and ages informed to consume this product daily or the twice to three times per week. A single estimate that would represent the average for both genders was $2.34(\mathrm{SD}=0.04)$, and for all the age ranges was $2.66(\mathrm{SD}=0.04)$, suggesting moderate consumption. The results revealed that the product is adequate as vehicle for iron fortification, based on the consumption aspect and the attitude of the researched group as potential consumers.

Keywords: school lunch, cookie, consumption frequency

\section{Introdução}

Entre as estratégias de intervenção definidas para a prevenção e o combate à deficiência de ferro e anemia ferropriva, a fortificação de alimentos de consumo expressivo foi considerada por pesquisadores da Organização Mundial de Saúde como a forma mais efetiva, segura e de menor custo, uma vez que pode atingir grande número de pessoas e ser utilizada por longo período de tempo (LOTFI, 1996; REILLY, 1996; CARDOSO \& PENTEADO, 1994).

A associação entre carência de ferro com ou sem anemia e as alterações no rendimento escolar de crianças e adolescentes tem sido descrita por vários pesquisadores, bem como tem sido recomendada a adoção de medidas que contribuam para prevenir e reduzir as repercussões da deficiência de ferro nesta população, utilizando a estrutura do programa de alimentação escolar (SILVA, 1996).

Para que um alimento seja eleito como veículo para a fortificação deve reunir características tais como: ser consumido basicamente por todas as pessoas da população alvo; o consumo per capita deve ser estável e uniforme; o alimento enriquecido deve manter estabilidade sob condições padrões de armazenamento e uso; os nutrientes adicionados devem ser fisiologicamente disponíveis e sem risco de ingestão excessiva; a fortificação não deve alterar as características sensoriais do produto, bem como não afetar o preço do mesmo sendo viável para o processo industrial (SILVA, 2000; RAUNHARDT \& BOWLEY, 1996; HURRELL, 1997).

No Brasil, a farinha de trigo e produtos correlatos, a farinha de milho, açúcar e arroz são alimentos indicados para ações de abrangência nacional por constituírem importante fonte de energia para a população (DARNTON-HILL, 1999).

De acordo com dados do Sindicato de massas e biscoitos do Estado de São Paulo - SIMABESP, 1999, o mercado de biscoitos no Brasil registrou aumento de vendas em torno de $80 \%$ no período de 1993 a 1998. Este mercado tem sido visto como promissor, tendo como público alvo principalmente as crianças e os adolescentes. O consumo médio per capita/ano é de 5,9 Kg e se aproxima da média mundial, com amplo interesse do setor industrial em oferecer produtos com maior valor nutritivo visando à complementação da alimentação básica (SIMABESP, 1999; BNDES, 2003).

Existem dois tipos de massas de biscoitos, duras e moles. A diferença reside na quantidade de água requerida para a elaboração da massa. Massas duras contêm maior teor de água e relativamente baixo teor de gordura (aspecto importante para fins de fortificação com ferro) e açúcar, incluindo-se entre estes os crackers e um grupo conhecido como semidoces ou doces-duros como os biscoitos "Maria" (MANLEY, 1989).

Biscoitos semidoces duros são comumente consumidos no Brasil, Canadá, Inglaterra e em diversos países da Europa. No Brasil, este tipo de biscoito é conhecido comercialmente com os nomes de Maria, Maizena, Manteiga, Leite e outros, dependendo do tipo de ingredientes utilizados (VITTI, 1988). Os biscoitos semidoces duros juntamente com biscoitos tipo crackers são designados básicos, totalizando aproximadamente $30 \%$ dos biscoitos comercializados no país (SIMABESP, 1999).

Uma das maneiras utilizadas para classificar os dados de freqüência de consumo consiste na agregação 
dos indivíduos em categorias de consumos elevado, médio ou baixo de um dado alimento, em geral correspondendo a consumo diário, ocasional e raro respectivamente, como exemplificado por Gibson, 1990.

Experiências com biscoitos fortificados com ferro e vitaminas, de acordo com Van Stuijvenberg et al, 1999, revelaram bons resultados na recuperação e prevenção de anemia em escolares, com resultados positivos também nas avaliações de desenvolvimento cognitivo.

Face ao exposto, a presente pesquisa objetivou conhecer a freqüência de consumo de biscoitos "Maria" por escolares do município de Fortaleza, CE, visando à eleição deste produto comumente identificado nos cardápios do Programa Nacional de Alimentação Escolar - PNAE, para fortificação com ferro, bem como selecionar escolares para a avaliação sensorial do produto fortificado.

\section{Metodologia}

A pesquisa foi realizada entre os dias 20 e 30 de novembro de 2000 , tendo como amostra alunos do ensino fundamental matriculados em 18 escolas da rede municipal de Fortaleza, CE. A seleção dos alunos foi realizada obedecendo a um plano amostral estratificado, no qual os estratos foram definidos pelas seis secretarias regionais às quais as escolas estavam vinculadas. Foram consideradas unidades primárias de seleção classes de $2^{\mathrm{a}}$ a $8^{\mathrm{a}}$ séries do Ensino Fundamental. Para participar da pesquisa foram sorteadas 18 escolas em um universo de 352 escolas municipais, sendo 3 escolas de cada secretaria regional, assim como 3 classes de cada escola sorteada. O plano amostral baseou-se nos dados cadastrais fornecidos pela Secretaria Municipal de Desenvolvimento Social da Prefeitura Municipal de Fortaleza, CE (SMDS, 2000).

Foram aplicados questionários em sala de aula para os alunos das classes amostradas obtendo-se informações sobre idade e gênero dos mesmos e os dados de freqüência de consumo de biscoitos Maria, os quais foram categorizados em itens representativos da freqüência de consumo (diário, intermediário, raro e nulo). Para o cálculo da freqüência média de consumo informada pelos escolares, neste estudo, atribuiu-se uma ponderação para os itens de freqüência de: i) 4 - diariamente (consumo elevado); ii) 3 - duas a três vezes por semana (consumo moderado); iii) $2-$ uma a duas vezes por mês (consumo baixo); iv) 1- uma a duas vezes por semestre (consumo raro);v) 0 nunca (consumo nulo).

Foi realizada análise descritiva dos dados de consumo de acordo com o gênero, a idade e a regional à qual as escolas pertenciam aplicando-se o teste Quiquadrado para verificação de associações. Calculouse também a freqüência de consumo (médio) para as referidas variáveis por meio de modelo linear geral descrito por Neter \& Wasserman, 1974, aplicando-se o teste estatístico de hipóteses com adoção do nível de significância de 5\%.

Para a análise estatística foram usados os recursos computacionais SPSS, versão 7.5 e Excel (1997).

\section{Resultados e discussão}

Caracterização dos alunos de acordo com o gênero, a idade e as regionais às quais as escolas eram vinculadas.

A caracterização dos alunos que participaram da pesquisa, com relação ao gênero está apresentada na Tabela 1.

Tabela 1. Distribuição dos alunos de acordo com o gênero. Fortaleza, CE, 2000.

\begin{tabular}{lcc}
\hline Gênero & Número de alunos (n) & Percentual \\
\hline Feminino & 371 & 51,00 \\
Masculino & 350 & 48,10 \\
Não responderam & 6 & 0,90 \\
\hline TOTAL & 727 & 100,00 \\
\hline
\end{tabular}

Entre os alunos pesquisados predominaram escolares do gênero feminino, sendo de $5,7 \%$ o percentual de diferença entre os gêneros. Embora no plano experimental para este estudo, não fosse possível estabelecer previamente o equilíbrio entre os gêneros dos participantes, a diferença relativamente pequena revelou-se favorável tendo em vista que é desejável o balanceamento de gêneros na composição de consumidores para testes de aceitabilidade de produtos, de acordo com Meilgaard (1988), uma vez que um dos objetivos foi avaliar a adequação da amostra de escolares sorteados para testes sensoriais com biscoitos fortificados. O percentual de $0,90 \%$ dos escolares pesquisados não informou o gênero, sendo mantidos na amostra pelo fato de terem informado a 
freqüência de consumo de biscoitos "Maria" e que participariam dos testes sensoriais do produto fortificado.

Para a análise dos dados de caracterização dos escolares por idade adotou-se a classificação apresentada na Tabela 2 que representou limites de idade esperados para o ensino fundamental, $1^{a}$ a $4^{a}$ séries e $5^{a}$ a $8^{a}$ séries respectivamente.

Tabela 2. Distribuição dos alunos de acordo com a faixa etária. Fortaleza, CE, 2000.

\begin{tabular}{lcc}
\hline Faixa Etária & Número de alunos (n) & Percentual \\
\hline$\leq 10$ anos & 104 & 14,30 \\
$\geq 11$ anos & 614 & 84,50 \\
Não responderam & 9 & 1,20 \\
\hline TOTAL & 727 & 100,00 \\
\hline
\end{tabular}

A média de idade observada foi de 13 anos $(\mathrm{DP}=0,08)$, com a menor idade encontrada de 7 anos e a maior de 18 anos. Para a idade, 1,2\% dos pesquisados não forneceram a informação, sendo mantidos na amostra por terem informado a freqüência de consumo e pelo fato de que as mesmas turmas amostradas participariam em etapa posterior de testes sensoriais com o produto fortificado.

Do total de escolares pesquisados, $26,7 \%$ pertenciam às classes referentes às primeiras séries do ensino fundamental ( $2^{\mathrm{a}}, 3^{\mathrm{a}}, 4^{\mathrm{a}}$ séries), e $73,35 \%$ eram alunos oriundos das séries mais avançadas do ensino fundamental ( $5^{\mathrm{a}}$ a $8^{\mathrm{a}}$ séries) indicando uma provável não correspondência entre séries e idade esperada para a série, no grupo de escolares pesquisados.

De acordo com a localização geográfica, as escolas municipais de Fortaleza, CE, encontram-se sob a coordenação de seis Secretarias Executivas, denominadas Regionais e identificadas por número na Tabela 3 por meio da qual foi demonstrada a distribuição dos alunos, em relação à regional a qual sua escola vinculava-se.

Para compor a amostra de escolares, todas as regionais foram representadas, tendo a Regional IV o maior número de alunos participantes e a Regional I o menor número deles. A variação da representatividade numérica de escolares de cada regional ocorreu em função da variação do número de alunos efetivamente presentes na data de aplicação dos questionários, por turmas definidas para a amostra, em relação ao esperado.

Tabela 3. Distribuição de alunos por secretaria regional. Fortaleza, CE, 2000.

\begin{tabular}{lcc}
\hline Faixa Etária & Número de alunos (n) & Percentual \\
\hline I & 101 & 13,90 \\
II & 93 & 12,80 \\
III & 137 & 18,90 \\
IV & 141 & 19,40 \\
V & 126 & 17,30 \\
VI & 129 & 17,70 \\
\hline TOTAL & 727 & 100,00 \\
\hline
\end{tabular}

Freqüência de consumo de Biscoitos "Maria" pelos escolares de acordo com o gênero.

Os dados de freqüência de consumo de biscoitos "Maria" segundo o gênero foram apresentados na Tabela 4.

Para ambos os gêneros observou-se uma tendência expressiva de consumo do produto. Entre os escolares, $63,10 \%$ do gênero feminino e $59,71 \%$ do gênero masculino informaram consumir biscoitos "Maria" diariamente ou 2 a 3 vezes por semana, expressando uma freqüência de consumo considerada moderada a elevada para aproximadamente $60 \%$ dos pesquisados de ambos os sexos.

Freqüencia de consumo pelos escolares segundo a idade

$\mathrm{Na}$ tabela 5 foram reunidos os dados de freqüência de consumo de biscoitos "Maria" pelos escolares por faixa etária.

Entre os escolares houve predominância de 63, $50 \%$ de crianças com idade máxima de 10 anos e 61,20 $\%$ de alunos acima desta faixa etária que informaram consumir biscoitos "Maria" diariamente ou 2 a 3 vezes por semana, o que reflete um consumo classificado como moderado a elevado.

Avaliando-se a freqüência de consumo diária e semanal para ambos os gêneros, $61,43 \%$, e faixas etárias, $61,56 \%$, constatou-se uma maior tendência 
de consumo pelo segmento estudado em relação a consumidores adultos na mesma região, para os quais Neumann et al., 2000 observaram uma freqüência de consumo diária ou semanal de $32 \%$, em estudo laboratorial de aceitação de biscoitos "Maria".
Os resultados de freqüência de consumo por idade neste estudo estão de acordo com tendências reveladas por pesquisas de mercado que têm nas crianças e adolescentes os maiores consumidores de biscoitos em geral (SIMABESP, 1999).

Tabela 4. Freqüência do consumo de biscoitos "Maria" de acordo com o gênero. Fortaleza, CE, 2000

\begin{tabular}{lcccccc}
\hline \multirow{2}{*}{ Gênero } & \multicolumn{3}{c}{ Freqüencia de consumo } \\
\cline { 2 - 7 } & Diariamente & $\begin{array}{c}2 \text { a } 3 \text { vezes } \\
\text { por semana }\end{array}$ & $\begin{array}{c}1 \text { a } 2 \text { vezes } \\
\text { no mês }\end{array}$ & $\begin{array}{c}1 \text { a } 2 \text { vezes } \\
\text { no semestre }\end{array}$ & Nunca & Total \\
\hline Feminino & 103 & 131 & 76 & 41 & 20 & 371 \\
Masculino & $(14,28 \%)$ & $(18,17 \%)$ & $(10,55 \%)$ & $(5,69 \%)$ & $(2,77 \%)$ & $(51,46 \%)$ \\
Total & 81 & 128 & 89 & 32 & 20 & 350 \\
& $(11,23 \%)$ & $(17,75 \%)$ & $(12,34 \%)$ & $(4,45 \%)$ & $(2,77 \%)$ & $(48,54 \%)$ \\
& 184 & 259 & 165 & 73 & 40 & 721 \\
$(25,51 \%)$ & $(35,92 \%)$ & $(22,89 \%)$ & $(10,14 \%)$ & $(5,54 \%)$ & $(100,00 \%)$
\end{tabular}

Tabela 5. Freqüência de consumo de biscoitos "Maria" dos alunos por idade. Fortaleza, CE, 2000.

\begin{tabular}{lcccccc}
\hline & \multicolumn{5}{c}{ Freqüencia de consumo } \\
\cline { 2 - 7 } Faixa Etária & Diariamente & $\begin{array}{c}2 \text { a } 3 \text { vezes } \\
\text { por semana }\end{array}$ & $\begin{array}{c}1 \text { a 2 vezes } \\
\text { no mês }\end{array}$ & $\begin{array}{c}1 \text { a } 2 \text { vezes } \\
\text { no semestre }\end{array}$ & Nunca & Total \\
\hline$\leq 10$ ANOS & 29 & 37 & 21 & 9 & 8 & 104 \\
& $(4,04 \%)$ & $(5,15 \%)$ & $(2,92 \%)$ & $(1,25 \%)$ & $(1,12 \%)$ & $(14,50 \%)$ \\
$\geq 11$ ANOS & 155 & 221 & 143 & 64 & 31 & 614 \\
TOTAL & $(21,59 \%)$ & $(30,77 \%)$ & $(19,92 \%)$ & $(8,91 \%)$ & $(4,32 \%)$ & $(85,50 \%)$ \\
& 184 & 258 & 164 & 73 & 39 & 718 \\
$(25,63 \%)$ & $(35,93 \%)$ & $(22,84 \%)$ & $(10,16 \%)$ & $(5,44 \%)$ & $(100,00 \%)$
\end{tabular}

Tabela 6. Freqüência do consumo de biscoitos "Maria” pelos escolares por Secretaria Regional. Fortaleza, CE, 2000.

\begin{tabular}{lcccccc}
\hline & \multicolumn{5}{c}{ Freqüencia de consumo } \\
\cline { 2 - 7 } Regional & Diariamente & $\begin{array}{c}2 \text { a } 3 \text { vezes } \\
\text { por semana }\end{array}$ & $\begin{array}{c}1 \text { a 2 vezes } \\
\text { no mês }\end{array}$ & $\begin{array}{c}1 \text { a } 2 \text { vezes } \\
\text { no semestre }\end{array}$ & Nunca & Total \\
\hline I & 21 & 30 & 29 & 12 & 9 & 101 \\
II & $(2,89 \%)$ & $(4,13 \%)$ & $(3,99 \%)$ & $(1,65 \%)$ & $(1,24 \%)$ & $(13,90 \%)$ \\
& 17 & 35 & 32 & 5 & 4 & 93 \\
III & $(2,34 \%)$ & $(4,81 \%)$ & $(4,40 \%)$ & $(0,69 \%)$ & $(0,55 \%)$ & $(12,80 \%)$ \\
& 38 & 46 & 36 & 12 & 5 & 137 \\
IV & $(5,23 \%)$ & $(6,33 \%)$ & $(4,95 \%)$ & $(1,65 \%)$ & $(0,69 \%)$ & $(18,90 \%)$ \\
& 30 & 50 & 28 & 17 & 16 & 141 \\
V & $(4,13 \%)$ & $(6,88 \%)$ & $(3,85 \%)$ & $(2,34 \%)$ & $(2,20 \%)$ & $(19,40 \%)$ \\
& 38 & 49 & 21 & 13 & 5 & 126 \\
VI & $(5,23 \%)$ & $(6,74 \%)$ & $(2,89 \%)$ & $(1,79 \%)$ & $(0,69 \%)$ & $(17,30 \%)$ \\
& 41 & 52 & 20 & 14 & 2 & 129 \\
TOTAL & $(5,64 \%)$ & $(7,15 \%)$ & $(2,75 \%)$ & $(1,93 \%)$ & $(0,27 \%)$ & $(17,70 \%)$ \\
& 185 & 262 & 166 & $73 \%)$ & 41 & 727 \\
& $(25,45 \%)$ & $(36,04 \%)$ & $(22,83 \%)$ & $(10,04 \%)$ & $(5,64 \%)$ & $(100,00 \%)$ \\
\hline
\end{tabular}


Para todas as regionais, foi de $61,49 \%$ a freqüência de consumo diário e semanal referida pelos escolares. Os maiores percentuais de freqüência de consumo diário e semanal foram observados para as regionais VI, $72,09 \%$, e V, $69,04 \%$, sendo o menor percentual, $50,49 \%$, encontrado para a regional I.

\section{Análise inferencial da frequiència de consumo de biscoitos "Maria" pelos escolares}

A análise inferencial foi realizada considerandose os dados de duas maneiras. A primeira na forma original de freqüências, como apresentados nas Tabelas 4,5 e 6 , e a segunda a partir da criação de um escore de freqüência de consumo.

A análise dos dados na forma de freqüências foi realizada através da aplicação do teste de quiquadrado, com o objetivo de verificar a existência de associação entre gênero, idade e localização geográfica e a freqüência de consumo. Os valores do teste foram 4,1909 com 4 graus de liberdade $(p=$ 0,3808) para gênero, 2,0379 com 4 graus de liberdade $(\mathrm{p}=0,7288)$ para a idade e $41,8806 \mathrm{com} 20$ graus de liberdade $(p=0,0029)$ para localização geográfica, indicando somente a existência de associação entre localização geográfica e freqüência de consumo, ao nível de significância de $5 \%$.

Para a segunda forma de análise foi criado um escore de freqüência de consumo pelos escolares, atribuindo-se uma ponderação para os itens de freqüência de: i) 4 - diariamente (consumo elevado); ii) 3 - duas a três vezes por semana (consumo moderado); iii) 2 - uma a duas vezes por mês (consumo baixo); iv) 1- uma a duas vezes por semestre (consumo raro); v) 0 - nunca (consumo nulo).

Considerando-se os dados apresentados nas Tabelas 4, 5 e 6 foram obtidas estimativas para as médias do escore de freqüência de consumo por gênero: $2,37(\mathrm{DP}=0,06)$ para o gênero feminino e 2,31 ( $\mathrm{DP}=$ $0,06)$ para o gênero masculino. Realizou-se uma análise de variância para averiguação de igualdade entre estas médias não se rejeitando a hipótese de igualdade $(\mathrm{p}=$ 0,4247 ) ao nível de significância de 5\%. Confirmou-se, portanto não existir diferença significativa para o escore de freqüência de consumo médio, entre os gêneros. Uma estimativa única para representar a média é 2,34 $(\mathrm{DP}=0,04)$, interpretada em função da ponderação atribuída, como média sugestiva de consumo moderado.
Obtiveram-se também estimativas para os valores médios do escore de freqüência de consumo para os dois grupamentos etários: 2,67 $(\mathrm{DP}=0,11)$ para crianças com 10 anos ou menos e 2,66 (DP = 0,04) para crianças com 11 anos ou mais. Por meio de uma análise de variância com o objetivo de verificar se estas médias são estatisticamente iguais, não se rejeitou a hipótese de igualdade $(p=0,9193)$ ao nível de significância de 5\%. Assim, uma estimativa única para representar a média do escore de freqüência de consumo é 2,66 (DP= 0,04) para as duas faixas etárias, indicando a tendência de consumo moderado.

Para os dados de freqüência de consumo dos escolares de acordo com a regional, foram calculadas as médias em função da ponderação estabelecida, obtendo-se os valores descritos na Tabela 7 , representativos das médias do escore de freqüência de consumo por regional.

Para a comparação do consumo médio das regionais foi realizada uma análise de variância, sendo rejeitada a hipótese de igualdade entre as médias $(\mathrm{p}=$ $0,0016)$ ao nível de significância de 5\%. A partir da realização de um teste de Tukey de comparação múltipla entre estas médias, verificou-se que somente a regional VI é que é efetivamente diferente das regionais I e IV.

Tabela 7. Escore médio de freqüência de consumo de biscoitos "Maria" por regional. Fortaleza, CE, 2000.

\begin{tabular}{lcc}
\hline Regional & $\begin{array}{c}\text { Freqüência Média } \\
\text { de consumo }\end{array}$ & $\begin{array}{c}\text { Desvio } \\
\text { Padrão }\end{array}$ \\
\hline I & $2,42^{\mathrm{a}}$ & 0,11 \\
II & $2,60^{\mathrm{ab}}$ & 0,10 \\
III & $2,73^{\mathrm{ab}}$ & 0,09 \\
IV & $2,43^{\mathrm{a}}$ & 0,10 \\
V & $2,81^{\mathrm{ab}}$ & 0,09 \\
VI & $2,90^{\mathrm{b}}$ & 0,08 \\
\hline
\end{tabular}

$\mathrm{Na}$ coluna, médias com letras iguais não diferem significativamente entre si $(p>0,05)$.

\section{Conclusões}

A análise dos dados de freqüência de consumo, por gênero, idade e regionais, revelou uma tendência de consumo moderado a elevado de biscoitos "Maria" 
pelos escolares pesquisados. Estes resultados confirmaram que o grupo amostrado representa consumidores potenciais de biscoitos tipo Maria, portanto, uma população que estaria apta a avaliar as formulações de biscoitos "Maria" fortificadas com ferro, por meio de testes sensoriais afetivos.

Uma das considerações relevantes para a seleção de alimentos veículo para fortificação com ferro é baseada no consumo expressivo e estável do alimento pela população alvo. Desta forma, os resultados obtidos para a freqüência de consumo indicaram ser adequada a escolha deste produto, sob o aspecto do consumo, como alimento veículo a ser fortificado com ferro, destinado aos escolares integrantes da amostra da pesquisa.

\section{Referências Bibliográficas}

BANCO NACIONAL DE DESENVOLVIMENTO ECONÔMICO E SOCIAL - BNDES Setorial. Cadeia Produtiva do Trigo, Rio de Janeiro, n.18, p. 193-220, 2003. Disponível em $<$ www.simabesp.com.br $>$. Acesso em 20 de maio de 2004.

CARDOSO, M. A. ; PENTEAdO, M.V.C. Intervenções Nutricionais na Anemia Ferropriva. Cad. de Saúde Pública, São Paulo, v 10, n. 2, p. 231 $-240,1994$.

DARNTON-HILL. et al. Iron and folate fortification in Americas to prevent and control micronutrient malnutrition: An analysis. Nutrition Reviews, v. 57, p.25-31, 1999.

GIBSON, R.S. Food consumption of individuals. In: Principles of nutritional assessment. New York: Oxford University Press, cap. 3, p. 37-53, 1990.

HURRELL, RF. Bioavailability of iron. European. J. Clin. Nutr. S1, 1997.

LOTFI, M. et al. Micronutrient Fortification of Food: current pratices, research and opportunites. Canadá: Research Centre and International Agriculture Centre, 1996. 107 p.

MANLEY, D.J.R. Tecnologia de La Indústria Galletera. Espanha: Editorial Acribia, 1989. 483 p.
MEILGAARD, M. et al.Sensory Evaluation Techniques. 2 ed. Flórida: CRC Press, 1988. 281p.

NETER, J.; WASSERMAN, W. Applied Linear Statistical Models. Illinois: Richard D. Irwin, INC. 1974.

NEUMANN, D. et al. Estudo de aceitação de Biscoitos Maria. In: Congresso Brasileiro de Ciência e Tecnologia de Alimentos, 17, 2000, Fortaleza. Anais. Fortaleza, CE, 2000. v.4, p.3242.

RAUNHARDT, O.; BOWLEY, A. Mandatory food enrichment. Nutriview n1. p. 1- 44, 1996. Supplement.

REILLY, C. Too much of a good thing? The problem of trace element fortification of foods. Trends in Food Science and Technology, v. 7, p.139-142, 1996.

SILVA, M. V. A trajetória do programa de merenda escolar (1954-1994) e o estado nutricional de crianças brasileiras. Cadernos de Nutrição- SBAN, São Paulo, v.11, p. 31-49. 1996.

SILVA, K. L. Critérios econômicos para a escolha de produtos a fortificar, ILSI Brasil - Enriquecimento e restauração de alimentos com micronutrientes - uma proposta para o Brasil, 2000.

Sindicato de Massas Alimentícias e Biscoitos no Estado de São Paulo-SIMABESP. Aumenta o consumo de massas no Brasil. Revista OESP Alimentação, São Paulo, n 26, 1999.

Secretaria Municipal de Desenvolvimento Social SMDS- Prefeitura Municipal de Fortaleza. Dados das escolas, séries, turno e número de alunos das regionais do município de Fortaleza. 2000.

VAN STUIJVENBERG, M.E. et al. Effect of iron, iodine and beta carotene fortified biscuits on the micronutrient status of primary school children: a randomized controlled trial. Am J Clin Nut. v. 69, p. 497-503,1999.

VITTI, P. et al. Tecnologia de Biscoitos. Manual Técnico. n. 1, Campinas, ITAL 1988. 86 p. 